\title{
Caracterização física e química da casca e polpa de jambo vermelho (Syzygium malaccensis, (L.) Merryl \& Perry)
}

\author{
Physical and chemical characterization of malay red-apple \\ (Syzygium malaccensis, (L.) Merryl \& Perry) Skin and pulp
}

\author{
Ivanilda Maria AUGUSTA ${ }^{1}$, Josane Maria RESENDE ${ }^{2 *}$, Soraia Vilela BORGES 3 , \\ Maria Cristina Antun MAIA ${ }^{4}$, Maria Antonieta Peixoto Gimenes COUTO ${ }^{4}$
}

\section{Resumo}

O objetivo do trabalho foi avaliar as propriedades físicas e químicas, respectivamente da polpa e da casca do jambo vermelho (Syzygium malaccensis) para possível aproveitamento na produção de novos produtos. Frutos de jambeiro maduros apresentaram rendimento médio em polpa de 75,69\%, umidade de $84,57 \mathrm{~g}$ de água. $100 \mathrm{~g}^{-1}$ de polpa e relação DL/DT de 1,24, forma levemente elíptica ou oval; conferindo boas características para industrialização. A casca corresponde a 8,05\% da polpa do fruto, é rica em fibras $\left(9,34 \mathrm{~g} .100 \mathrm{~g}{ }^{-1}\right)$, em vitamina C (292,59 mg.100 g $\mathrm{g}^{-1}$ ) e em antocianinas (300,54 mg.100 g-1 de casca). O pH de 3,5 classifica-a como muito ácida, favorecendo seu uso na fabricação de geleias para acidificação e em sucos para aumentar o rendimento. A casca de jambo é uma boa fonte de fibras, carboidratos e vitamina C, fornecendo 37 e $45 \%$ da IA de fibras e de carboidratos, respectivamente, e $390 \%$ da IDR de vitamina C. O jambo apresentou características físicas adequadas para a industrialização na forma de polpa congelada, sucos, doce em massa e em compota, geleias, néctares e sorvetes e a casca, propriedades físicas e químicas que permitem seu reaproveitamento como antioxidante, corante natural em alimentos e no enriquecimento de dietas em função dos altos teores de vitamina $\mathrm{C}$ e anticianinas.

Palavras-chave: Syzygium malaccensis; jambo vermelho; aproveitamento da polpa e da casca; caracterização física e química.

\begin{abstract}
The objective of this study was to analyze the physical and chemical properties of the pulp and skin of the malay red-apple (Syzygium malaccensis),respectively, for possible use in the production of new products. Ripe malay red-apple presented a mean percent pulp yield of $75.69 \%$, moisture content of $84.57 \mathrm{~g} .100 \mathrm{~g}^{-1}$ of pulp, Longitudinal Diameter (DL)/Transversal Diameter (DT) ratio of 1,24, and slightly elliptical or oval shape; thus presenting good characteristics for industrialization. The skin accounted for $8.05 \%$ of the fruit pulp and was rich in fiber (9.34 g.100 g $\left.\mathrm{g}^{-1}\right)$, vitamin C (292.59 mg.100 g-1), and in anthocyanins (300.54 mg.100 g-1 of skin). The pH value was 3.5 allowing for its classification as a very acid food and suitable for use in the manufacture of jams for acidification as well as in juices to increase the yield. The malay red-apple skin is a good source of fiber, carbohydrate and vitamin C providing 37 and $45 \%$ of the adequate Intake (AI) of fiber and carbohydrate, respectively, and $390 \%$ of the RDI vitamin C. The malay red-apple presents physical characteristics that make it adequate for industrialization as frozen pulp, juice, preserves, jams, nectar, and ice cream, and its skin shows physical and chemical properties that allow for its use as a food antioxidant and colorant and also to enrich diets.

Keywords: Syzygium malaccensis; malay red-apple; use of pulp and skin; physical and chemical properties.
\end{abstract}

\section{Introdução}

O Jambo vermelho (Syzygium malaccensis, (L.) Merryl \& Perry) pode ser consumido in natura, em forma de compotas, doce em massa, geleias, licores e aguardente e ainda pode ser utilizado para a produção de corante e antioxidante natural para uso em vários segmentos da indústria. Os frutos do jambeiro apresentam cor vermelho escuro, levemente adocicado, exalando aroma de rosas, persistente e bastante agradável ao olfato. As características físicas dos frutos, como cor, tamanho, número de sementes, quantidade de polpa e o conteúdo de água, podem influenciar no seu consumo, tanto ao natural quanto pela indústria. A caracterização física e química é importante para avaliação da qualidade, classificação tecnológica do fruto, fornecendo informações seguras para avaliação do valor nutricional, do rendimento, das operações de processamento e da vida útil do produto.

Segundo Kurosawa (2004), o jambo contém vitaminas A, B1, B12, proteínas, antocianinas, além de cálcio, ferro e fósforo. De acordo com Costa et al. (2006), a polpa, que constitui $84 \%$ do fruto, apresenta ${ }^{\circ}$ Brix de 6,8 e acidez de $0,4 \mathrm{mg} .100 \mathrm{~g}^{-1}$ de polpa, no final da maturação. $\mathrm{O}$ fruto tem uma aparência atrativa em função da cor vermelha intensa e da forma, é apreciado pelo

\footnotetext{
Recebido para publicação em 6/8/2008

Aceito para publicação em 8/7/2009 (003743)

Pós-graduação em Tecnologia de Processos Químicos e Bioquímicos, Escola de Química, Universidade Federal do Rio do Janeiro - UFRJ, E-mail: ivanildamariaa@yahoo.com.br

2 Departamento de Tecnologia de Alimentos, Instituto de Tecnologia, Universidade Federal Rural do Rio de Janeiro - UFRRJ, Rod. BR 465, km 7, CEP 23890-000,

Seropédica - RJ, Brasil,E-mail: jo_resende@oi.com.br

3 Departamento de Ciência dos Alimentos, Universidade Federal de Lavras - UFLA

${ }^{4}$ Departamento de Engenharia Bioquímica, Escola de Química, Universidade Federal do Rio de Janeiro - UFRJ

${ }^{*}$ A quem a correspondência deve ser enviada
} 
seu sabor e aroma exóticos e possui propriedades aromáticas interessantes que o favorece como agente flavorizante em alimentos e bebidas. Ocorre um grande desperdício de jambo na época da safra, em virtude da elevada produção de frutos por árvore, do curto período da safra e da reduzida vida útil do fruto in natura. Da produção ao consumo, a magnitude das perdas de jambo é considerável (ALMEIDA et al., 2005).

Poucos dados estão disponíveis na literatura sobre a composição nutricional da polpa e casca de jambo vermelho, tornando-se necessária sua caracterização para um melhor aproveitamento do fruto, evitando desperdícios e tornando possível a melhoria da qualidade nutricional dos cardápios pela incorporação de farinhas nutritivas, pelo aproveitamento do seu extrato para produção de geleias e ainda para extração de corantes naturais (LIMA et al., 2002). Além disso, o conhecimento da composição nutricional de frutos permite: a população consumir os nutrientes de acordo com a Ingestão Diária Recomendada (IDR); o desenvolvimento de pesquisas que estabeleçam uma relação entre dieta e doenças uma vez que frutos e hortaliças são fontes importantes de nutrientes (vitaminas, minerais e flavonoides) na dieta humana; além de um melhor planejamento agrícola e das indústrias de alimentos (GONDIM et al., 2005).

O pouco conhecimento sobre a composição física e química dos frutos desta espécie motivou seu estudo que, além de apresentar grande potencial econômico, poderá subsidiar o cultivo e a seleção adequada, visando o seu aproveitamento na indústria de alimentos.

Com o objetivo de incentivar o aproveitamento do jambo vermelho na safra e oferecer uma alternativa nutritiva de dieta de baixo custo, frutos e cascas de jambo foram analisados quanto à composição física e química respectivamente para possível aproveitamento na produção de novos produtos.

\section{Material e métodos}

Foram utilizados jambos vermelhos produzidos no município de Seropédica, Estado do Rio de Janeiro no período de abril a maio de 2007. Os frutos foram colhidos manualmente no estádio maduro, levando em consideração a coloração da casca vermelho intenso e as características sensoriais de maturação (gosto e aroma), foram acondicionados em contentores de plástico para evitar injúrias mecânicas e transportados ao laboratório de Tecnologia de Alimentos da Universidade Federal Rural do Rio de Janeiro.

Após a seleção para eliminar os frutos danificados, estes foram lavados em água corrente e sanitizados com cloro na concentração de $200 \mathrm{mg} \cdot \mathrm{L}^{-1}$ durante 10 minutos. Em seguida, foram secos em temperatura ambiente. Antes do descascamento, foram selecionados aleatoriamente 50 frutos para as análises físicas de umidade ( $\mathrm{g}$ de água. $100 \mathrm{~g}^{-1}$ de polpa) por meio de secagem em estufa a vácuo, modelo Luferco Instrumentos Científicos, com circulação de ar a $75{ }^{\circ} \mathrm{C}$, segundo técnica do Instituto Adolfo Lutz (2005); do diâmetro transversal (cm), avaliado na parte mais larga do fruto, e longitudinal $(\mathrm{cm})$ determinados com auxílio de um paquímetro digital Mitutoyo stainless hardened; da massa dos frutos (g), massa das sementes (g) e massa das cascas (g) determinadas por meio de pesagem em de balança semianalítica modelo Máster-Fisatom. O rendimento em polpa e em casca foi obtido pela relação percentual entre a massa do fruto inteiro e de suas respectivas estruturas.

Para as análises físicas e químicas da casca, os frutos foram separados em 10 lotes e descascados manualmente com auxílio de faca inox. Amostras de cerca de $300 \mathrm{~g}$ de cascas de cada lote foram acondicionadas em sacos de polietileno $(27 \times 29 \mathrm{~cm})$, com $0,08 \mathrm{~mm}$ de espessura, atóxico, inodoro, incolor, hermético e armazenadas em freezer $\mathrm{a}-18^{\circ} \mathrm{C}$.

Para as análises, as amostras foram descongeladas em geladeira e trituradas em multiprocessador doméstico Arno, modelo magiclean chrome para as seguintes determinações: $\mathrm{pH}$ por meio de $\mathrm{pHmetro}$ digital Quimis, modelo Q.400A; teor de Acidez Total Titulável (ATT) em ácido cítrico determinado por titulação com bureta, segundo técnica do Instituto Adolfo Lutz (2005); Sólidos Solúveis Totais (SST) determinados por refratometria, utilizando refratômetro portátil de campo, modelo RT-30ATC e os resultados expressos em ${ }^{\circ} \mathrm{Brix}$; teor de umidade ( $\mathrm{g}$ de água. $100 \mathrm{~g}^{-1}$ de casca) por meio de secagem em estufa a vácuo, modelo Luferco Instrumentos Científicos, com circulação de ar a $75{ }^{\circ} \mathrm{C}$, segundo técnica do Instituto Adolfo Lutz (2005); teores de açúcares redutores (mg de glicose. $100 \mathrm{~g}^{-1}$ de casca) e não redutores (mg de açúcares totais. $100 \mathrm{~g}^{-1}$ de casca) por titulação pelo método de Fehling (INSTITUTO ADOLFO LUTZ, 2005); teor de vitamina C total (mg ácido ascórbico $100 \mathrm{~g}^{-1}$ de casca), de acordo com método titulométrico com 2,6- diclorofenolindofenol (DCFI) até o ponto de viragem, segundo Cunniff (1997); teor de lipídios totais (g. $100 \mathrm{~g}^{-1}$ de casca); proteínas (g.100 g $\mathrm{g}^{-1}$ de casca); fibras totais (g.100 g ${ }^{-1}$ de casca); cinzas ( $\mathrm{g} .100 \mathrm{~g}^{-1}$ de casca), segundo técnica recomendada pelo Instituto Adolfo Lutz (2005); teor de cálcio (mg.100 g-1 de casca) de acordo com Silva (1990); e teor de antocianinas totais (mg.100 g $\mathrm{g}^{-1}$ de casca), determinado segundo metodologia de Giust e Wrolstad (2002). O valor calórico foi calculado utilizando-se dos seguintes fatores de conversão de Atwater: $9 \mathrm{kcal}$ por g de lipídios, $4 \mathrm{kcal}$ por $\mathrm{g}$ de proteínas e $4 \mathrm{kcal}$ por g de carboidratos (DE ANGELIS, 1977). As determinações foram realizadas em triplicatas 72 horas após o congelamento do primeiro lote de cascas. Foi feita análise de estatística descritiva em que se obtiveram as médias dos três lotes e os desvios padrão.

\section{Resultados e discussão}

A polpa do Jambo caracteriza-se por apresentar alto teor de umidade $84,57 \mathrm{~g}$ de água. $100 \mathrm{~g}^{-1}$ de polpa (Tabela 1 ), enquadrando-se na classe dos frutos carnosos e suculentos, sendo esta uma das características comuns de frutos da família Myrtaceae, como o jambolão (87,75 g de água) (LAGO; GOMES; SILVA, 2006), cambuci $(88,8 \mathrm{~g})$, jabuticaba $(87,85 \mathrm{~g})$, uvaia $(85,53 \mathrm{~g})$, pitanga $(90,47 \mathrm{~g})$ e goiaba vermelha $(85,81 \mathrm{~g})$ (VALLILO et al., 2005).

Os dados do Diâmetro Longitudinal (DL) e Transversal (DT) e a relação entre eles (DL/DT) (Tabela 1) indicam que os frutos de $S$. malaccensis têm forma levemente elíptica ou 
Tabela 1. Avaliação das características físicas da polpa de jambo vermelho.

\begin{tabular}{lc}
\hline \multicolumn{1}{c}{ Características } & Valores médios $^{*}$ \\
\hline Diâmetro Transversal (DT) $(\mathrm{cm})$ & $4,40 \pm 0,41$ \\
Diâmetro Longitudinal (DL) (cm) & $5,44 \pm 0,58$ \\
Relação DL/DT & $1,24 \pm 0,49$ \\
Massa do fruto (g) & $39,16 \pm 1,30$ \\
Massa da polpa (g) & $29,64 \pm 1,25$ \\
Massa da semente (g) & $6,95 \pm 0,96$ \\
Massa da casca (g) & $2,57 \pm 0,28$ \\
Rendimento da polpa (\%) & 75,69 \\
Rendimento da casca (\%) & 8,05 \\
Umidade (g.100 g ${ }^{-1}$ de polpa) & $84,57 \pm 0,21$ \\
\hline
\end{tabular}

${ }^{*}$ Média de 50 frutos.

oval (DL/DT > 1), característica dessa espécie botânica. Para a fabricação de doces em calda ou glaciados, em que a aparência do produto final é primordial, normalmente, dá-se preferência a frutos com uniformidade de formato levemente arredondado ou oblongo (DL/DT = 1) (ANDRADE; ARAGÃO; FERREIRA, 1993). Embora não apresente forma oblonga, o jambo tem um formato exótico que, associado à cor vermelho intenso, $\mathrm{o}$ torna muito atrativo aos olhos do consumidor, favorecendo o seu aproveitamento para produção de doces glaciados e em compota.

Os diâmetros transversais e longitudinais estão relacionados com o tamanho e a forma do fruto, enquanto a massa dos frutos, das cascas e das sementes está relacionada com o rendimento do produto, tornando-se fatores importantes no estabelecimento do ponto de maturação, da viabilidade econômica para a industrialização, além do dimensionamento de embalagens (RESENDE, 2007; VALLILO et al., 2005).

Pela análise dos resultados apresentados na Tabela 1, observa-se que o rendimento da polpa corresponde a 75,69\% do fruto, dos $24,31 \%$ restantes, a casca contribui com $10,57 \%$ e as sementes com 13,74\%. Esses dados são importantes porque permitem calcular o rendimento do fruto durante o processamento para obtenção de polpa para doces em massa, em compota e ou polpa congelada. Segundo Andrade, Aragão e Ferreira (1993), a massa fresca do fruto e os rendimentos de suas partes interferem na eficiência dos processos industriais para fabricação de doces, exigindo uma adequada classificação ou separação prévia dos frutos por tamanho ou massa.

Assim, frutos de jambeiro apresentam boas características para industrialização na forma de doces, polpa congelada, sucos, geleias, néctares e sorvetes, devido ao percentual médio de rendimento em polpa em frutos maduros de 75,69\%, embora Costa et al. (2006) afirme que o rendimento da polpa pode chegar a $84 \%$ do fruto. O rendimento em casca corresponde a $8,05 \%$ da polpa do fruto (Tabela 1). Essas cascas, na maioria das vezes descartadas como resíduos, podem ser reaproveitadas para obtenção de farinhas usadas no enriquecimento de alimentos ou ainda na obtenção de corantes usados na indústria de alimentos.
Com relação à casca do jambo (Tabela 2), verifica-se que tem alto teor de fibras (9,34 g.100 g $\left.\mathrm{g}^{-1}\right)$. Quando comparada às cascas de outros frutos, consideradas ricas em fibras, como o abacaxi $\left(3,89\right.$ g. $\left.100 \mathrm{~g}^{-1}\right)$, maracujá $\left(4,33\right.$ g. $\left.100 \mathrm{~g}^{-1}\right)$, tangerina $\left(10,38 \mathrm{~g} .100 \mathrm{~g}^{-1}\right)$

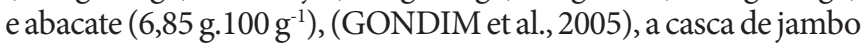
apresenta-se como uma das mais ricas em fibras.

As quantidades de vitamina C (292,59 mg.100 g $\left.\mathrm{g}^{-1}\right)$ e antocianinas (300,54 mg. $\left.100 \mathrm{~g}^{-1}\right)$ (Tabela 2) são elevadas e, como na maioria dos frutos da família Myrtaceae, concentram-se em maior proporção na casca. Em pitanga-roxa, por exemplo, o teor de antocianinas na polpa e casca foi de 26 e $420 \mathrm{mg} .100 \mathrm{~g}^{-1}$ do fruto, respectivamente (LIMA; MÉLO; LIMA, 2002). Ao comparar o teor antocianinas encontrado na casca de jambo vermelho (300,54 mg. $100 \mathrm{~g}^{-1}$ de casca) com os encontrados em outras cascas de frutos como de jabuticabas (314 mg.100 g ${ }^{-1}$ de casca), jambolão (386 mg. $100 \mathrm{~g}^{-1}$ de casca), uva (227 mg.100 g $\mathrm{g}^{-1}$ de casca) (TERCI, 2004) e açaí (263 mg.100 g $\mathrm{g}^{-1}$ de casca) (BOBBIO et al., 2000), observa-se que a casca de jambo é uma fonte rica em antocianinas, uma vez que são considerados frutos ricos em antocianinas aqueles que apresentam mais do que $2 \mathrm{mg}$ de antocianinas $\mathrm{g}^{-1}$ do fruto (MACHEIX; FLEURIET; BILLOT, 1990). Portanto, sob esse aspecto, todos estes frutos vêm despertando a atenção de produtores e consumidores em função dos benefícios que proporcionam ao organismo, devido à presença de elevado teor de compostos fenólicos com poder antioxidante (LAGO; GOMES; SILVA, 2006).

Em relação ao teor vitamina C (292,59 mg. $\left.100 \mathrm{~g}^{-1}\right)$, a casca de jambo vermelho supera os valores encontrados para outros frutos da família Myrtaceae, que apresentam em média teor de vitamina C variando de 12 a $80 \mathrm{mg}$ de ácido ascórbico. $100 \mathrm{~g}^{-1}$ de polpa para jabuticaba e goiaba vermelha, respectivamente (VALLILO et al., 2005). Assim a casca de jambo vermelho pode tanto ser usada para obtenção de corantes como de antioxidantes para uso em alimentos.

As cascas são ácidas ( $\mathrm{pH}=3,5)$ (Tabela 2), favorecendo os processos de industrialização na forma de doces, geleias e sucos. Sendo possível o seu aproveitamento na fabricação de geleias como forma de acidificação para obtenção de géis adequados e para o enriquecimento do produto e sucos, uma vez que na indústria de sucos, o alto teor de acidez provoca elevada diluição do produto e, por conseguinte, maior rendimento final (ANDRADE; ARAGÃO; FERREIRA, 1993). O pH da casca de jambo $(3,5)$ encontra-se dentro da faixa estipulada para frutos da família Myrtaceae de 2,54 a 4,09 para pêra do campo e jambolão, respectivamente (VALLILO et al., 2005; LIMA; MÉLO; LIMA, 2002).

$\mathrm{Na}$ composição química de $S$. malaccensis, verificou-se a presença de alto teor de lipídios (4,51 g.100 g-1 de casca) que, somado ao teor de carboidratos $\left(59,25 \mathrm{~g} .100 \mathrm{~g}^{-1}\right.$ de casca), indica valor energético de $312,07 \mathrm{kcal} 100 \mathrm{~g}^{-1}$, sendo que o conteúdo de proteína $\left(8,62 \mathrm{~g} .100 \mathrm{~g}^{-1}\right.$ de casca) pouco contribui nesse sentido.

Com os resultados obtidos na Tabela 2, foi possível calcular as contribuições percentuais da casca de jambo em relação à Ingestão Diária Recomendada (IDR) e a Ingestão Adequada (IA), (INSTITUTE OF MEDICINE, 2005) para cada nutriente analisado. Estes percentuais, considerando-se os requerimentos nutricionais para um adulto mulher de 19 a 50 anos, encontramse na Tabela 3 . 
Tabela 2. Avaliação das características físicas e químicas da casca de jambo vermelho.

\begin{tabular}{|c|c|}
\hline Características & Valores médios \\
\hline Umidade (g.100 $\mathrm{g}^{-1}$ de casca) & $14,11 \pm 0,4$ \\
\hline Sólidos Solúveis Totais ( ${ }^{\circ}$ Brix) & $3,00 \pm 0,01$ \\
\hline $\mathrm{pH}$ & $3,50 \pm 0,02$ \\
\hline Acidez total titulável (ATT) (g ac. cítrico. $100 \mathrm{~g}^{-1}$ de casca) & $0,60 \pm 0,11$ \\
\hline Açúcares redutores (g.glicose. $100 \mathrm{~g} \mathrm{~g}^{-1}$ de casca) & $3,04 \pm 0,4$ \\
\hline Açúcares não redutores (g aç. Totais. $100 \mathrm{~g}^{-1}$ de casca) & $\mathrm{ND}^{* *}$ \\
\hline Vitamina C (mg.100 $\mathrm{g}^{-1}$ de casca) & $292,59 \pm 0,80$ \\
\hline Antocianina (mg.100 g ${ }^{-1}$ de casca) & $300,54 \pm 0,45$ \\
\hline Carboidratos (g.100 g-1 de casca) & $59,25 \pm 0,15$ \\
\hline Proteínas (g.100 g ge casca) & $8,62 \pm 0,23$ \\
\hline Lipídios (g.100 g-1 de casca) & $4,51 \pm 0,10$ \\
\hline Fibras (g.100 g ${ }^{-1}$ de casca) & $9,34 \pm 0,16$ \\
\hline Cinzas (g. $100 \mathrm{~g}^{-1}$ de casca) & $4,17 \pm 0,35$ \\
\hline Cálcio (mg.100 g-1 de casca) & $0,36 \pm 0,72$ \\
\hline Valor calórico total (kcal.100 g $\mathrm{g}^{-1}$ de casca) & $312,07 \pm 0,90$ \\
\hline
\end{tabular}

Tabela 3. Ingestão Diária Recomendada (IDR), Ingestão Adequada (IA) e percentual da IDR e IA fornecidos pela casca de jambo para um adulto mulher de 19 a 50 anos.

\begin{tabular}{lcc}
\hline Características & $\mathrm{IDR}^{(1)}$ & \% IDR e IA em relação a $100 \mathrm{~g}$ de casca \\
\hline Proteínas & $46(\mathrm{~g} / \mathrm{dia})$ & 18 \\
Lipídios & $35(\mathrm{~g} / \mathrm{dia})$ & 12 \\
Fibras & $25-35(\mathrm{~g} / \mathrm{dia})$ & $37^{*}$ \\
Carboidratos & $130(\mathrm{~g} / \mathrm{dia})$ & 45 \\
Cálcio & $1000(\mathrm{mg} / \mathrm{dia})$ & 0,036 \\
Vitamina C & $75(\mathrm{mg} / \mathrm{dia})$ & 390 \\
\hline
\end{tabular}

(1) INSTITUTE OF MEDICINE. Food and nutrition board. dietary reference intakes for energy, carbohydrate, fiber, fat, fatty acids, cholesterol, protein, and amino acids (Macronutrients). Washignton, D.C: The National Academy Press, 2005; *Ingestão Adequada (IA)

A casca de jambo pode ser considerada uma boa fonte de fibras e vitamina C, fornecendo $37 \%$ da IA para fibras e $390 \%$ da IDR de vitamina C. Entretanto, para o cálcio, a contribuição é de apenas $0,036 \%$, sendo a casca considerada uma fonte pobre em cálcio. Embora possua altos teores de carboidratos, lipídios e proteínas em relação a outros frutos da família Myrtaceae, estes contribuem com apenas 45, 12 e 18\% da IDR, respectivamente, sendo os carboidratos os principais responsáveis pelo alto valor energético da casca $\left(312,07 \mathrm{kcal} .100 \mathrm{~g}^{-1}\right)$. Embora rico em energia, a casca de jambo contribui com apenas 18\% para o requerimento estimado de energia para um adulto mulher de 19 ou mais anos, sedentário ( $1.737 \mathrm{kcal})$.

A vitamina $\mathrm{C}$, devido ao seu alto poder redutor, proporciona proteção contra a oxidação descontrolada no meio aquoso da célula, reduzindo significativamente os efeitos adversos produzidos por espécies reativas, como aquelas do oxigênio e nitrogênio (AMAYA-FARFAN; DOMENE; PADOVANI, 2001). Na literatura, é considerável o volume de informações indicando que o excesso de espécies reativas de oxigênio e nitrogênio ocasiona danos às células normais e, portanto, causam disfunção celular e doenças neurodegenerativas centrais (BLOT; LI; TAYLOR, 1993; US NATIONAL..., 2000).
Em relação às fibras, segundo dados levantados pelo Instituto Brasileiro de Geografia e Estatística (2004), o consumo pela população brasileira é baixo, acompanhado pela elevada ingestão de gorduras e proteínas (NEUTZLING et al., 2007). O estudo apontou que $61,2 \%$ da população consomem fibras abaixo do recomendado (INSTITUTO BRASILEIRO..., 2004; MATTOS; MARTINS, 2000). A recomendação da Associação Dietética Americana (2002) é similar à da Organização Mundial da Saúde (OMS): a de que a ingestão de fibras alimentares para adultos seja de 20 a $35 \mathrm{~g} /$ dia.

A baixa ingestão de fibras está associada principalmente ao baixo consumo de frutos, vegetais e cereais integrais (AMAYAFARFAN; DOMENE; PADOVANI, 2001). A Organização Mundial da Saúde, em conjunto com a Organização das Nações Unidas para Agricultura e Alimentação (INSTITUTE OF MEDICINE, 2005; NISHIDA et al., 2004), sugeriu que a ingestão diária de mais de $400 \mathrm{~g}$ de frutos e vegetais é necessária para que se atinja a Ingestão Adequada (IA). Assim a quantidade de fibra encontrada na casca de jambo $\left(9,34 \mathrm{~g} .100 \mathrm{~g}^{-1}\right)$ atende $37 \%$ da IA recomendada para um adulto (Tabela 3 ), tornando-a adequada para o aproveitamento no complemento de dietas para a redução de peso e suplementação de fibras para regularização das funções intestinais. Estimular o consumo habitual de hortaliças, frutos, cereais e grãos integrais pode representar uma alternativa para aumentar o consumo de fibras e promover melhoria para a saúde humana (BRASIL, 2005).

\section{Conclusões}

A polpa do jambo apresentou características adequadas para o consumo ao natural e industrialização devido aos seus atributos de qualidade, como alto rendimento em polpa e forma levemente elíptica ou oval que lhe confere um formato exótico. A casca apresentou composição química adequada para enriquecer alimentos ou como fonte para extração de corante e antioxidante, usados na indústria de alimentos devido ao alto teor de carboidratos, o baixo valor energético, a elevada acidez e as altas concentrações de ácido ascórbico, antocianinas e fibra alimentar.

\section{Referências bibliográficas}

ALMEIDA, J. B. O. et al. Uso de leveduras de vinhos de frutas tropicais na alimentação humana. Brazilian Journal of Food Technology, p. 65-69, 2005. 5a SPIAL.

AMAYA-FARFAN, J.; DOMENE, S. M. A.; PADOVANI, R. M. DRI: Síntese comentada das novas propostas sobre recomendações nutricionais para antioxidantes. Revista de Nutrição, v. 14, n. 1, p. 71-78, 2001.

ANDRADE, J. S.; ARAGÃO, C. G.; FERREIRA, S. A. N. Caracterização física e química dos frutos de Araçá-Pêra (Psidium acutangulum) D. C. Acta Amazônica, v. 23, n. 2-3, p. 213- 217, 1993.

ASSOCIAÇÃO DIETÉTICA AMERICANA. Health implications of dietary fiber. Journal of the American Dietetic Association, v. 102, p. 993-1000. 2002. Disponível em: <http://www.eatright.org/cps/ rde/xchg/ada/hs.xsl/advocacy_adar2_0702_ENU_HTML_(Draft). htm>. Acesso em: 13 de maio 2008.

BLOT, W. J., LI, J-Y., TAYLOR, P. R. Nutrition intervention trials in Linxian, China: Supplementation with specific vitamin/mineral 
combinations, cancer incidence and disease-specific mortality in the general population. Journal of the National Cancer Institute, Bethesda, v. 85, p. 1483-1492, 1993.

BOBBIO, F. O. et al. Identificação e quantificação das antocianinas dos frutos do açaizeiro (Etuerpe oleracea) Mart.). Ciência e Tecnologia de Alimentos, v. 20, n. 3, p. 388-390, 2000.

BRASIL. Ministério da Saúde. Secretaria de Atenção à Saúde. Coordenação-Geral da Política de Alimentação e Nutrição. Guia alimentar para a população brasileira: promovendo a alimentação saudável. Brasília: Ministério da Saúde, 2005. 236p. Disponível em: $<$ http://www.nutritotal.com.br/publicacoes/?acao=bu\&id=155\&ca tegoria=7>. Acesso em: 13 de maio 2008.

COSTA, R. S. et al. Aspectos morfológicos e influência do tamanho da semente na germinação do jambo-vermelho. Revista Brasileira de Fruticultura, v. 28, n. 1, p. 117-120, 2006.

CUNNIF, P. Official Methods of Analiysis of AOAC International. 16. ed. Gaithersburg: AOAC-International, 1997. v. 2.

DE ANGELIS, R. C. Fisiologia da nutrição: fundamentos para nutrição e desnutrição. São Paulo: EDART/EDUSP, 1977. v. 1, p. 44.

GIUST, M. M.; WROLSTAD, R. E. Characterization and Measurement of Anthocyanins by UV-Visible Spectroscopy. In: WROLSTAD, R. E. et al. Current protocols in food analytical chemistry. New York: John Wiley \& Sons, 2002.

GONDIM, J. A. M. et al. Composição centesimal e de minerais em casca de frutas. Ciência e Tecnologia de Alimentos, v. 25, n. 4, p. 825-827, 2005.

INSTITUTE OF MEDICINE. Food and nutrition board. dietary reference intakes for energy, carbohydrate, fiber, fat, fatty acids, cholesterol, protein, and amino acids (Macronutrients). Washignton, D.C: The National Academy Press, 2005. Disponível em: $<$ http://books.nap.edu/openbook.php?record_ id=10490\&page=339>. Acesso em: 27 abr. 2008 .

INSTITUTO ADOLFO LUTZ. Normas analíticas do Instituto Adolfo Lutz. Métodos físicos e químicos para análise de alimentos. 4. ed. São Paulo, 2005. 1018 p.

INSTITUTO BRASILEIRO DE GEOGRAFIA E ESTATÍSTICA IBGE. Pesquisa do orçamento familiar: 2002-2003. Análise da disponibilidade domiciliar de alimentos e do estado nutricional no Brasil. Rio de Janeiro, 2004. Disponível em: <http://www.ibge.gov. br/home/estatistica/populacao/condicaodevida/pof/2002analise/ pof2002analise.pdf>. Acesso em: 02 abr. 2008.
KUROSAWA, C. Jambo-Vermelho. Disponível em: < http:/redeglobo. globo.com/cgibin/globoruralmontar_texto_ult.pl?controle $=2040>$ Acesso em: 04 maio 2008.

LAGO, E. S.; GOMES, E.; SILVA, R. Produção de geléia de jambolão (Syzygium cumini Lamarck): processamento, parâmetros físico químicos e avaliação sensorial. Ciência e Tecnologia de Alimentos, v. 26, n. 4 , p. $847-852,2006$.

LIMA, E. D. P. A. et al. Caracterização física e química dos frutos da umbu-cajazeira (Spondias spp) em cinco estádios de maturação, da polpa congelada e néctar. Revista Brasileira de Fruticultura, v. 24, n. 2, p. 338-343, 2002.

LIMA, V. L. A. G.; MÉLO, E. A.; LIMA, D. E. S. Fenólicos e carotenóides totais em pitanga. Scientia Agrícola, v. 59, n. 3, p. 447-450, 2002.

MACHEIX, J. J.; FLEURIET, A.; BILLOT, J. Fruits Phenolics. Boca Raton: CRC Press, 1990.

MATTOS, L. L.; MARTINS, I. S. Dietary fiber consumption in an adult population. Revista de Saúde Pública, v. 34, n. 1, p. 50-55, 2000.

NEUTZLING, M. B. et al. Frequency of high-fat and low-fiber diets among adolescents. Revista de Saúde Pública, São Paulo, v. 41, n. 3, p. 336-342, 2007.

NISHIDA, C. et al. The Joint WHO/FAO Expert Consultation on diet, nutrition and the prevention of chronic diseases: process, product and policy implications. Public Health Nutrition, v. 7, n. 1A, p. 245-250, 2004. Disponível em: <http://journals.cambridge.org/ action/displayAbstract $?$ fromPage $=$ online $\&$ aid $=569940 \#>$. Acesso em: 22 abr. 2008.

RESENDE, J. M. Revestimentos biodegradáveis para conservação do coco 'ANÃO VERDE'. 2007. 221 f. Tese (Doutorado em Engenharia Agrícola)-Universidade Estadual de Campinas, Campinas, SP, 2007.

SILVA, D. J. Análise de alimentos- Métodos químicos e biológicos. Viçosa: UFV/ Impressa Universitária, 1990. 165 p.

TERCI, D. B. L. Aplicações analíticas e didáticas de antocianinas extraídas de frutas. 2004. 213 f. Tese (Doutorado em Química)Universidade Estadual de Campinas, Campinas, SP, 2004.

US NATIONAL ACADEMY OF SCIENCES (USA). Dietary Reference Intakes for vitamin $C$, vitamin $E$, selenium and carotenoids. Washington DC: National Academic Press, 2000. 506 p.

VALLILO, M. I. et al. Características físicas e químicas dos frutos do cambucizeiro (Campomanesia phaea). Revista Brasileira de Fruticultura, v. 27, n. 2, p. 241-244, 2005. 